\title{
Evolution of AF ablation strategies-Role of PVI?
}

\section{Introduction}

Atrial fibrillation (AF) is the most common clinical arrhythmia and its prevalence in the United States is expected to reach 6-12 million by the year 2050 [1]. A few decades ago, it was believed that AF was caused by a set of random and disorganized activations in the atria. Moe et al. [2,3] in 1964 first proposed his 'multiple wavelet hypothesis' according to which, new wavelets form after the initial wave break resulting in the multiple wavelet fibrillation. These wavelets are short lived and can either disintegrate after colliding with another wavelet or a non-conducting boundary, or fractionate in to multiple daughter wavelets. The second hypothesis put forward by Lewis in 1925 [4] and then by Gurvich in 1975 was the 'mother rotor hypothesis,' in which AF is maintained by a single rapid reentrant circuit of excitation [5]. This theory has been studied in detail by Jalife's group in large animal models and simulations, as the predominant mechanism for AF [6,7]. These two proposed mechanisms have been shown to appear in isolation and also simultaneously, resulting in a much more complex pattern of AF [8]. More recently in 2010, Allessie proposed 'the double layer hypothesis' adding a third dimension to the complexity of AF in which endo-epicardial dissociation plays a major role in maintaining AF $[9,10]$.

In a majority of the AF cases, antiarrhythmic drugs are not effective and hence catheter ablation has become main line of therapy in certain subgroups of patients. Several studies have demonstrated various multiple ablation strategies with limited efficacy. Pulmonary vein isolation (PVI) has proven to be efficacious for paroxysmal AF, but its efficacy in persistent AF remains low [11]. In 1998, Haïssaguerre et al. [12] in his seminal paper showed that pulmonary veins (PV) triggers are critical for the initiation of $\mathrm{AF}$ and PVI can suppress occurrence of AF. In this study, $94 \%$ of the ectopic triggers originated from
PVs resulted in AF. These observations Uma Mahesh R Avula* and Elaine Wan made PVI the cornerstone in the invasive Department of Medicine Cardiology, Columbia University, treatment of AF and was quickly adapted by ${ }^{\text {New York, NY 10032, USA }}$ the electrophysiologists around the world umamahes@med.umich.edu and became the preferred ablation strategy Submitted: 07 September 2017 $[13,14]$. However, long term follow up after Accepted: 25 September 2017 ablation, showed higher recurrence rates of AF especially in patients with persistent AF with success rates as low as $30 \%$ in the $3-5$ year follow up $[15,16]$. These meager successes have shifted the focus on elucidating the mechanisms in which $\mathrm{AF}$ occurs and perpetuates despite PVI and how operators can target atrial substrates for a successful ablation.

Nademanee et al. [17] in 2004, introduced the concept of ablating complex fractionated atrial electrograms (CFAE). This approach restored sinus rhythm in $95 \%$ of the cases at 12 months follow up. However, the success rate was limited on a large scale due to the complexity of mapping fractionated electrograms which may be highly operator dependent and the results were not reproducible [18]. In 2012, Narayan et al. [19] introduced clinically a new method to identify and ablate AF sources which were thought to be the source of rotational activity in the heart that caused AF. Haïssaguerre et al. [20] published similar results in 2014 . As various and combinations of ablation strategies increased success rates, performing solely PVI for AF ablation has remained a topic of debate. Recent multicentre randomized study (STAR AF II) [21] showed that PVI alone is sufficient in AF ablation and that additional lines may decrease long term success. However, peri-procedural AF termination was low in the group where only PVI was performed. Recently, Seitz et al. [22] showed there was increased effectiveness of AF ablation by targeting electrogram spatio-temporal dispersion sites and compared to the validation set in which patients underwent conventional PVI for paroxysmal AF and PVI+additional line and fractionated electrogram ablation for 
persistent AF: 18 month follow up showed increased success rate in these patients as compared to the validation set $(95 \%$ vs $63 \%)$. These results demonstrated that spatiotemporal dispersion of electrograms is the main source of AF drivers and that a patient specific ablation strategy targeting dispersion only can be used. This leaves the pulmonary veins intact and thus decreasing the potential complications from PVI such as PV stenosis. Jadidi et al. [23] confirmed this approach of spatio-temporal dispersion ablation in their recently published article.

Multiple studies showed that pulmonary vein triggers are not the only mechanisms responsible for AF. PVI alone is not enough in these patients and several studies have shown improved outcome with additional substrate ablation such as ablating ganglion plexi [24], ligament of Marshall ablation [25], CFAE [17], focal impulse or rotor modulation (FIRM) [19], dominant frequency ablation [26], and more recently 'Substrate Ablation Guided by High Density Mapping in Atrial Fibrillation (SUBSTRATE HD)' [22]. There is an evolution of new ablation strategies as we further understand the mechanisms of the AF initiation and perpetuation. These new approaches of ablation demonstrate superiority of substrate based ablation over PVI, especially in patients with persistent AF. Though the results are encouraging, more randomized studies are needed to validate these recent developments in the ablation field.

\section{References}

1. Miyasaka Y, Barnes ME, Gersh BJ, et al. Secular trends in incidence of atrial fibrillation in Olmsted county, minnesota, 1980 to 2000 , and implications on the projections for future prevalence. Circulation. 114: 119-125 (2006).

2. Moe GK, Rheinboldt WC, Abildskov JA. A computer model of atrial fibrillation. Am. Heart. J. 67: 200-220 (1964).

3. Moe GK. Evidence for reentry as a mechanism of cardiac arrhythmias. Rev. Physiol. Biochem. Pharmacol. 72: 55-81 (1975).

4. Lewis $\mathrm{T}$. The mechanism and graphic registration of the heart beat shaw. Physiol. 8: 9 (1925)

5. Gurvich N. The main principles of cardiac defibrillation. Medicine. 9: 8 (1975)

6. Samie FH, Berenfeld O, Anumonwo J, et al. Rectification of the background potassium current: A determinant of rotor dynamics in ventricular fibrillation. Circulation. Res. 89: 1216-1223 (2001).

7. Zaitsev AV, Berenfeld O, Mironov SF, Jalife J, Pertsov AM. Distribution of excitation frequencies on the epicardial and endocardial surfaces of fibrillating ventricular wall of the sheep heart. Circulation. Res. 86: 408-417 (2000).

8. Lee S, Sahadevan J, Khrestian CM, Durand DM, Waldo AL. High density mapping of atrial fibrillation during vagal nerve stimulation in the canine heart: Restudying the moe hypothesis. J. Cardiovascul. Electrophysiol. 24: 328-335 (2013).

9. Allessie MA, de Groot NM, Houben RP, et al. Electropathological substrate of long-standing persistent atrial fibrillation in patients with structural heart disease. Circulation: Arrhyth. Electrophysiol. 3: 606-615 (2010).

10. de Groot N, Vander DL, Yaksh A, et al. Direct proof of endoepicardial asynchrony of the atrial wall during atrial fibrillation in humans. Circulation: Arrhyth. Electrophysiol. 9: e003648 (2016).

11. Nakahara S, Hori Y, Nishiyama N, et al. Influence of the left atrial contact areas on fixed low-voltage zones during atrial fibrillation and sinus rhythm in persistent atrial fibrillation. $J$. Cardiovascul. Electrophysiol. 8: 9 (2017).

12. Haissaguerre M, Jaïs P, Shah DC, et al. Spontaneous initiation of atrial fibrillation by ectopic beats originating in the pulmonary veins. N. Engl. J. Med. 339: 659-666 (1998).

13. Jais P, Weerasooriya R, Shah DC, et al. Ablation therapy for atrial fibrillation (af): Past, present and future. Cardiovascul. Res. 54: 337-346 (2002).

14. Oral H, Scharf C, Chugh A, et al. Catheter ablation for paroxysmal atrial fibrillation: Segmental pulmonary vein ostial ablation versus left atrial ablation. Circulation.108: 2355-2360 (2003).

15. Ouyang F, Tilz R, Chun J, et al. Long-term results of catheter ablation in paroxysmal atrial fibrillation: Lessons from a 5year follow-up. Circulation. 122: 2368-2377 (2010).

16. Sawhney N, Anousheh R, Chen WC, Narayan S, Feld GK Five-year outcomes after segmental pulmonary vein isolation for paroxysmal atrial fibrillation. Am. J. Cardiol. 104: 366-372 (2009).

17. Nademanee K, McKenzie J, Kosar E, et al. A new approach for catheter ablation of atrial fibrillation: Mapping of the electrophysiologic substrate. J. Am. Coll. Cardiol. 43: 2044-2053 (2004)

18. Adragão P, Carmo P, Cavaco D, et al. Relationship between rotors and complex fractionated electrograms in atrial fibrillation using a novel computational analysis. Rev. Portug. Cardiol. 36: 233-238 (2017).

19. Narayan SM, Krummen DE, Shivkumar K, Clopton P, Rappel WJ, Miller JM. Treatment of atrial fibrillation by the ablation of localized sources: Confirm (conventional ablation for atrial fibrillation with or without focal impulse and rotor modulation) trial. J. Am. Coll. Cardiol. 60: 628-636 (2012).

20. Haissaguerre M, Hocini M, Denis A, et al. Driver domains in persistent atrial fibrillation. Circulation. 113: 005421 (2014).

21. Verma A, Jiang CY, Betts TR, et al. Approaches to catheter ablation for persistent atrial fibrillation. N. Engl. J. Med. 372: 1812-1822 (2015).

22. Seitz J, Bars C, Théodore G, et al. Af ablation guided by spatiotemporal electrogram dispersion without pulmonary vein isolation: A wholly patient-tailored approach. J. Am. Coll. Cardiol. 69: 303-321 (2017).

23. Jadidi AS, Lehrmann H, Keyl C, et al. Ablation of persistent atrial fibrillation targeting low-voltage areas with selective activation characteristics. Circulation: Arrhyth. Electrophysiol. 9: e002962 (2016)

24. Pokushalov E, Romanov A, Artyomenko S, et al. Ganglionated plexi ablation for longstanding persistent atrial fibrillation. Europace. 12: 342-346 (2010).

25. Hwang C, Chen PS. Ligament of marshall: Why it is important for atrial fibrillation ablation. Heart. Rhythm. 6: S35-S40 (2009).

26. Kumagai K, Sakamoto T, Nakamura K, et al. Combined dominant frequency and complex fractionated atrial electrogram ablation after circumferential pulmonary vein isolation of atrial fibrillation. J. Cardiovascul. Electrophysiol. 24: 975-983 (2013). 